\title{
A PRIORI BOUND ON THE VELOCITY IN AXIALLY SYMMETRIC NAVIER-STOKES EQUATIONS
}

\author{
ZHEN LEI, ESTEBAN A NAVAS AND QI S. ZHANG
}

\begin{abstract}
Let $v$ be the velocity of Leray-Hopf solutions to the axially symmetric threedimensional Navier-Stokes equations. Under suitable conditions for initial values, we prove the following a priori bound

$$
|v(x, t)| \leq \frac{C|\ln r|^{1 / 2}}{r^{2}}, \quad 0<r \leq 1 / 2,
$$

where $r$ is the distance from $x$ to the $z$ axis, and $C$ is a constant depending only on the initial value.

This provides a pointwise upper bound (worst case scenario) for possible singularities while the recent papers [5] and [13] gave a lower bound. The gap is polynomial order 1 modulo a half $\log$ term.
\end{abstract}

\section{Contents}

1. Introduction

2. A priori bound for $\omega_{\theta}$

3. Velocity Bound, proof of Theorem 1.1

4. Appendix: a regularity criteria for $v_{z}$ only

Acknowledgements

References

\section{INTRODUCTION}

In this paper, we prove, under suitable initial condition, that the flow speed in the axially symmetric incompressible flow has an a priori bound which is proportional to the inverse square of the distance to the rotational axis, modulo a half logarithmic term. In order to present the result precisely, let us first recall the basic set ups. In Cartesian coordinates, the incompressible Navier-Stokes equations are

$$
\Delta v-(v \cdot \nabla) v-\nabla p-\partial_{t} v=0, \operatorname{div} v=0,
$$

where $v=\left(v_{1}(x, t), v_{2}(x, t), v_{3}(x, t)\right): \mathbb{R}^{3} \times[0, T] \rightarrow \mathbb{R}^{3}$ is the velocity field and $p=$ $p(x, t): \mathbb{R}^{3} \times[0, T] \rightarrow \mathbb{R}$ is the pressure. In cylindrical coordinates $r, \theta, z$ with $\left(x_{1}, x_{2}, x_{3}\right)=$

Date: Sept. 2013; revised Feb. 2015.

AMS Subject Classifications: 35Q30 and 35B07. 
$(r \cos \theta, r \sin \theta, z)$, axially symmetric solutions are of the form

$$
v(x, t)=v_{r}(r, z, t) \overrightarrow{e_{r}}+v_{\theta}(r, z, t) \overrightarrow{e_{\theta}}+v_{z}(r, z, t) \overrightarrow{e_{z}}
$$

The components $v_{r}, v_{\theta}, v_{z}$ are all independent of the angle of rotation $\theta$. Here $\overrightarrow{e_{r}}, \overrightarrow{e_{\theta}}, \overrightarrow{e_{z}}$ are the basis vectors for $\mathbb{R}^{3}$ given by

$$
\overrightarrow{e_{r}}=\left(\frac{x_{1}}{r}, \frac{x_{2}}{r}, 0\right), \overrightarrow{e_{\theta}}=\left(\frac{-x_{2}}{r}, \frac{x_{1}}{r}, 0\right), \overrightarrow{e_{z}}=(0,0,1)
$$

It is well known (see [4] for example) that $v_{r}, v_{z}$ and $v_{\theta}$ satisfy the equations

$$
\left\{\begin{array}{l}
\left(\Delta-\frac{1}{r^{2}}\right) v_{r}-(b \cdot \nabla) v_{r}+\frac{v_{\theta}^{2}}{r}-\frac{\partial p}{\partial r}-\frac{\partial v_{r}}{\partial t}=0, \\
\left(\Delta-\frac{1}{r^{2}}\right) v_{\theta}-(b \cdot \nabla) v_{\theta}-\frac{v_{\theta} v_{r}}{r}-\frac{\partial v_{\theta}}{\partial t}=0, \\
\Delta v_{z}-(b \cdot \nabla) v_{z}-\frac{\partial p}{\partial z}-\frac{\partial v_{z}}{\partial t}=0, \\
\frac{1}{r} \frac{\partial\left(r v_{r}\right)}{\partial r}+\frac{\partial v_{z}}{\partial z}=0,
\end{array}\right.
$$

where $b(x, t)=\left(v_{r}, 0, v_{z}\right)$ and the last equation is the divergence-free condition. Here, $\Delta$ is the cylindrical scalar Laplacian and $\nabla$ is the cylindrical gradient field:

$$
\Delta=\frac{\partial^{2}}{\partial r^{2}}+\frac{1}{r} \frac{\partial}{\partial r}+\frac{1}{r^{2}} \frac{\partial^{2}}{\partial \theta^{2}}+\frac{\partial^{2}}{\partial z^{2}}, \quad \nabla=\left(\frac{\partial}{\partial r}, \frac{1}{r} \frac{\partial}{\partial \theta}, \frac{\partial}{\partial z}\right) .
$$

Observe that the equation for $v_{\theta}$ does not explicitly depend on the pressure. Let $\Gamma=r v_{\theta}$, one sees that the function $\Gamma$ satisfies

$$
\Delta \Gamma-(b \cdot \nabla) \Gamma-\frac{2}{r} \frac{\partial \Gamma}{\partial r}-\frac{\partial \Gamma}{\partial t}=0, \operatorname{div} b=0 .
$$

Recall that the vorticity $\omega=\operatorname{curl} v$ for axially symmetric solutions

$$
\omega(x, t)=\omega_{r} \overrightarrow{e_{r}}+\omega_{\theta} \overrightarrow{e_{\theta}}+\omega_{z} \overrightarrow{e_{z}}
$$

is given by

$$
\omega_{r}=-\frac{\partial v_{\theta}}{\partial z}, \omega_{\theta}=\frac{\partial v_{r}}{\partial z}-\frac{\partial v_{z}}{\partial r}, \omega_{z}=\frac{\partial v_{\theta}}{\partial r}+\frac{v_{\theta}}{r} .
$$

The equations of vorticity $\omega=\operatorname{curl} v$ in cylindrical form are (again, see [4] for example):

$$
\left\{\begin{array}{l}
\left(\Delta-\frac{1}{r^{2}}\right) \omega_{r}-(b \cdot \nabla) \omega_{r}+\omega_{r} \frac{\partial v_{r}}{\partial r}+\omega_{z} \frac{\partial v_{r}}{\partial z}-\frac{\partial \omega_{r}}{\partial t}=0, \\
\left(\Delta-\frac{1}{r^{2}}\right) \omega_{\theta}-(b \cdot \nabla) \omega_{\theta}+2 \frac{v_{\theta}}{r} \frac{\partial v_{\theta}}{\partial z}+\omega_{\theta} \frac{v_{r}}{r}-\frac{\partial \omega_{\theta}}{\partial t}=0, \\
\Delta \omega_{z}-(b \cdot \nabla) \omega_{z}+\omega_{z} \frac{\partial v_{z}}{\partial z}+\omega_{r} \frac{\partial v_{z}}{\partial r}-\frac{\partial \omega_{z}}{\partial t}=0 .
\end{array}\right.
$$

Define $\Omega=\frac{\omega_{\theta}}{r}$, then we have that $\Omega$ satisfies

$$
\Delta \Omega-(b \cdot \nabla) \Omega+\frac{2}{r} \frac{\partial \Omega}{\partial r}-\frac{\partial \Omega}{\partial t}+\frac{2 v_{\theta}}{r^{2}} \frac{\partial v_{\theta}}{\partial z}=0, \operatorname{div} b=0
$$

If the swirl $v_{\theta}=0$, then it is known for long time (see O. A. Ladyzhenskaya [14, M. R. Uchoviskii and B. I. Yudovich [20]), that finite energy solutions to (1.1) are smooth for all time. See also the paper by S. Leonardi, J. Malek, J. Necas, and M. Pokorny [17]).

In the presence of swirl, it is not known in general if finite energy solutions blow up in finite time. However a lower bound for the possible blow up rate is known by the recent results of C.-C. Chen, R. M. Strain, T.-P.Tsai, and H.-T. Yau in [4], [5], G. Koch, N. 
Nadirashvili, G. Seregin, and V. Sverak in [13]. See also the work by G. Seregin and V. Sverak [18] for a localized version. These authors prove that if

$$
|v(x, t)| \leq \frac{C}{r}
$$

then solutions are smooth for all time. Here $C$ is any positive constant. Solutions satisfying this bound are often refereed to as type I solutions. One reason for this name is that the bound scales the same way as the reciprocal of the distance. Their result can be rephrased as: type I solutions are regular. See also the paper by two of us [15], [16] on further results in this direction. Without knowing if blow up happens in general, it is desirable to find an upper bound for the growth of velocity. It is expected that the solutions are smooth away from the axis, with certain growing bound when approaching the axis. Our Theorem 1.1 confirms this intuitive idea. Although it did not give the bound (1.5) which is required for smoothness, it reveals the exact gap between what we have and what we need.

This seems to be the first pointwise bound for the speed (velocity) for the axially symmetric Navier-Stokes equation. We mention that a less accurate a priori upper bound for the vorticity has been found in 1 .

The following are some notations to be frequently used. We use $x=\left(x_{1}, x_{2}, x_{3}\right)$ to denote a point in $\mathbb{R}^{3}$ for rectangular coordinates, and in the cylindrical system we use $r=\sqrt{x_{1}^{2}+x_{2}^{2}}, \theta=\tan ^{-1} \frac{x_{2}}{x_{1}}, z=x_{3}$. Also $x^{\prime}=\left(x_{1}, x_{2}, 0\right)$.

Here is the main result of the paper.

Theorem 1.1. Suppose $v$ is a smooth, axially symmetric solution of the three-dimensional Navier-Stokes equations in $\mathbb{R}^{3} \times(-T, 0)$ with initial data $v_{0}=v(\cdot,-T) \in L^{2}\left(\mathbb{R}^{3}\right)$. Assume further $r v_{0, \theta} \in L^{\infty}\left(\mathbb{R}^{3}\right)$ and let $R=\min \{1, \sqrt{T / 2}\}$.

Then for all $(x, t) \in \mathbb{R}^{3} \times\left(-R^{2}, 0\right)$, it holds

$$
\left|v_{r}(x, t)\right|+\left|v_{z}(x, t)\right| \leq \frac{C \sqrt{|\ln r|}}{r^{2}}, \quad 0<r \leq \min \{1 / 2, R\}
$$

Here $r$ is the distance from $x$ to the $z$ axis, and $C$ is a constant depending only on the initial data.

The proof of the theorem is based on the following pointwise bound on the vorticity.

Theorem 1.2. Suppose $v$ is a smooth, axially symmetric solution of the three-dimensional Navier-Stokes equations in $\mathbb{R}^{3} \times(-T, 0)$ with initial data $v_{0}=v(\cdot,-T) \in L^{2}\left(\mathbb{R}^{3}\right)$, and $\omega$ is the vorticity. Assume further, $r v_{0, \theta} \in L^{\infty}\left(\mathbb{R}^{3}\right)$ and let $R=\min \{1, \sqrt{T / 2}\}$. Then the following a priori estimate holds. 
There is a constant $C$, depending only on the initial data, such that the following holds for all $(x, t) \in \mathbb{R}^{3} \times\left(-R^{2}, 0\right)$ with $r=\left|x^{\prime}\right| \in\left(0, \min \left\{R, \frac{1}{2}\right\}\right)$ :

$$
\begin{aligned}
\left|\omega_{\theta}(x, t)\right| \leq \frac{C \ln (1 / r)}{r^{7 / 2}}\left[\sup _{s \in\left[t-r^{2}, t\right]}\left(\int_{B(x, 4 r)}\left(v_{r}^{2}+v_{z}^{2}\right)(y, s) d y\right)^{1 / 2}+r^{1 / 2}\left(\left\|r v_{0, \theta}\right\|_{L^{\infty}\left(\mathbb{R}^{3}\right)}+1\right)\right]^{2} & \\
\times & {\left[\left(\int_{t-r^{2}}^{t} \int_{B(x, 4 r)} \omega_{\theta}^{2}(y, s) d y d s\right)^{1 / 2}+r^{1 / 2}\left(\left\|r v_{0, \theta}\right\|_{L^{\infty}\left(\mathbb{R}^{3}\right)}+1\right)\right] . }
\end{aligned}
$$

Remark 1.1. We assume smoothness of the solution only for technical simplicity. One can use standard approximation methods to treat the weak solution case. In fact, if $v$ is a (suitable) Leray-Hopf solution, then it is smooth except possibly on the $z$ axis (c.f. [2]). Also, the bound on $\omega_{\theta}$ is scaling invariant. Similar bounds can also be proven for the other two components of the vorticity $\omega_{r}$ and $\omega_{z}$. But we will not do this here.

Here we mention a number of related papers on axially symmetric Navier-Stokes equations. J. Neustupa and M. Pokorny [9] proved that the regularity of one component (either $v_{r}$ or $v_{\theta}$ ) implies regularity of the other components of the solution. Also proving regularity is the work of Q. Jiu and Z. Xin [10] under an assumption of sufficiently small zero-dimension scaled norms. D. Chae and J. Lee [3] also proved regularity results assuming finiteness of another certain zero-dimensional integral. G. Tian and Z. Xin [19] constructed a family of singular axially symmetric solutions with singular initial data. T. Hou and C. Li 6] found a special class of global smooth solutions. See also a recent extension: T. Hou, Z. Lei and C. Li [8].

Let us outline the proof of the theorem. The starting point is the a priori bound for the rotational component of the velocity: $r\left|v_{\theta}(\cdot, t)\right| \in L^{\infty}$. A proof of this fact can be found in [3] Section 3, Proposition 1, for example. The first ingredient is the observation that the basic energy estimate is critical when localized in a dyadic ball which is away from the symmetric axis. This enables us to perform a kind of dimension reduction argument and apply two-dimensional Sobolev imbedding inequalities. The second ingredient is a new estimate on the oscillation of the angular stream function in a dyadic ball which is based on dimension reduction and the structure of the equations. Then we apply the standard Moser iteration method and two-dimensional Sobolev imbedding inequalities to get an upper bound for the quantity $\Omega=w_{\theta} / r$, based on the evolution equation of $\Omega$ in (1.4). The third ingredient is a novel use of the localized Biot-Savart law. We use axis-symmetry to show that $L^{2}$ integrals of velocity in small dyadic regions are smaller than usual. This fact and the a priori bound on $\omega^{\theta}$ implies the point-wise bound on $\left|v^{r}\right|+\left|v^{z}\right|$.

Throughout this paper we use $C$ to denote an absolute positive constant. When $C$ depends on $p$, we use the notation $C_{p}$. The meanings of $C$ and $C_{p}$ may change from line to line.

The remainder of the paper is organized as follows. In Section 2, we prove Theorem 1.2 the a priori bound on $\omega_{\theta}$. In Section 3 we prove Theorem 1.1. Some additional results are given in the appendix. 


\section{A PRIORI BOUND FOR $\omega_{\theta}$}

In this section, we will prove Theorem 1.2,

Let $(x, t)$ be the point in the statement of the theorem. For simplicity we take $t=0$ and $x_{3}=0$. During the proof, it is convenient to replace the three dimensional ball $B(x, 4 r)$ by comparable cylindrical type regions. The reason is that a cylindrical region has a fixed profile in the $e_{r}, e_{z}$ plane. This feature allows us to reduce much computations to 2 dimensional setting.

So let us introduce a few more notations. Let $R>0, S>0$, and $0<A<B$ be constants.

Denote

$$
C_{A R, B R}=\left\{\left(x_{1}, x_{2}, x_{3}\right): A R \leq r \leq B R, 0 \leq \theta \leq 2 \pi,\left|x_{3}\right| \leq B R\right\} \subset \mathbb{R}^{3}
$$

to be the hollowed out cylinder centered at the origin, with inner radius $A R$, outer radius $B R$, and height extending up and down $B R$ units for a total height of $2 B R$. If $R=1$, we will write $C_{A, B}$ in place of $C_{A 1, B 1}$.

Denote $P_{A R, B R, S R}$ to be the parabolic region

$$
P_{A R, B R, S R}=C_{A R, B R} \times\left(-S^{2} R^{2}, 0\right) .
$$

If $R=1$, we will use $P_{A, B, S}$ to denote $P_{A 1, B 1, S 1}$.

Proving the theorem is equivalent to finding priori bound for $\omega_{\theta}$ in the region $P_{\frac{k}{2}, 2 k, \frac{3 k}{4}}$ with $0<k<\min \{1, \sqrt{T / 2}\}$. We will use the scaling property of the Navier Stokes equation to shift the consideration to the cube $P_{\frac{1}{2}, 2, \frac{3}{4}}$. We recall that scaling of the equations now; the pair $(v(x, t), p(x, t))$ is a solution to the system, if and only if for any $k>0$ the re-scaled pair $(\widetilde{v}(x, t), \widetilde{p}(x, t))$ is also a solution, where $\widetilde{v}(x, t)=k v\left(k x, k^{2} t\right)$, $\widetilde{p}(x, t)=k^{2} p\left(k x, k^{2} t\right)$. Thus, if $(v, p)$ is a solution to the axially symmetric Navier-Stokes equations for $(x, t) \in P_{k, 4 k, k}$, then $(\widetilde{v}(\widetilde{x}, \widetilde{t}), \widetilde{p}(\widetilde{x}, \widetilde{t}))$ is a solution to the equation in the variables $\widetilde{x}=\frac{x}{k}, \widetilde{t}=\frac{t}{k^{2}}$ when $(\widetilde{x}, \widetilde{t}) \in P_{1,4,1}$. We note here how certain quantities scale or change due to the above. Here, $\mathrm{D}$ is any domain in $\mathbb{R}^{3}$ and $k D=\{x: x=k y, y \in D\}$ :

$$
\begin{gathered}
r=\sqrt{x_{1}^{2}+x_{2}^{2}}: \quad \widetilde{r}=\sqrt{\left(\frac{x_{1}}{k}\right)^{2}+\left(\frac{x_{2}}{k}\right)^{2}}=\frac{r}{k} \\
\|v(x, t)\|_{L^{2}\left(k D \times\left(-(k R)^{2}, 0\right)\right)}: \\
\|\widetilde{v}(\widetilde{x}, \widetilde{t})\|_{L^{2}\left(D \times\left(-R^{2}, 0\right)\right)}=\left(\int_{-R^{2}}^{0} \int_{D} \mid \widetilde{v}\left(\widetilde{x},\left.\widetilde{t}\right|^{2} d \widetilde{x} d \widetilde{t}\right)^{\frac{1}{2}}\right. \\
=\left(\int_{-(k R)^{2}}^{0} \int_{k D}|k v(x, t)|^{2} \frac{1}{k^{5}} d x d t\right)^{\frac{1}{2}}=\frac{1}{k^{\frac{3}{2}}}\|v(x, t)\|_{L^{2}\left(k D \times\left(-(k R)^{2}, 0\right)\right) .} \\
b(x, t)=\left(v_{r}, 0, v_{z}\right): \\
\widetilde{b}(x, t)=\left(k v_{r}\left(k x, k^{2} t\right), 0, k v_{z}\left(k x, k^{2} t\right)\right)=k b\left(k x, k^{2} t\right), \quad(x, t) \in P_{k, 4 k, k} \\
\Rightarrow \widetilde{b}(\widetilde{x}, \widetilde{t})=k b(x, t) . \\
\|b(x, t)\|_{L^{\infty}\left(-(k R)^{2}, 0 ; L^{2}(k D)\right)}:
\end{gathered}
$$




$$
\begin{aligned}
& \|\widetilde{b}(\widetilde{x}, \widetilde{t})\|_{L^{\infty}\left(-R^{2}, 0 ; L^{2}(D)\right)}=\sup _{-R^{2} \leq \widetilde{t}<0}\left(\int_{D}|\widetilde{b}(\widetilde{x}, \widetilde{t})|^{2} d \widetilde{x}\right)^{\frac{1}{2}} \\
& =\sup _{-(k R)^{2} \leq t<0}\left(\int_{k D}|k b(x, t)|^{2} \frac{1}{k^{3}} d x\right)^{\frac{1}{2}}=\frac{1}{k^{\frac{1}{2}}}\|b(x, t)\|_{L^{\infty}\left(-(k R)^{2}, 0 ; L^{2}(k D)\right)} . \\
& \omega(x, t): \quad \widetilde{\omega}(x, t)=k^{2} \omega\left(k x, k^{2} t\right),(x, t) \in P_{1,4,1} \Rightarrow \widetilde{\omega}(\widetilde{x}, \widetilde{t})=k^{2} \omega(x, t) \\
& \|\omega(x, t)\|_{L^{2}\left(k D \times\left(-(k R)^{2}, 0\right)\right)}: \\
& \mid \widetilde{\omega}(\widetilde{x}, \widetilde{t}) \|_{L^{2}\left(D \times\left(-R^{2}, 0\right)\right)}=\left(\int_{-R^{2}}^{0} \int_{D}|\widetilde{\omega}(\widetilde{x}, \widetilde{t})|^{2} d \widetilde{x} d \widetilde{t}\right)^{\frac{1}{2}} \\
& =\left(\int_{-(k R)^{2}}^{0} \int_{k D}\left|k^{2} \omega(x, t)\right|^{2} \frac{1}{k^{5}} d x d t\right)^{\frac{1}{2}}=\frac{1}{k^{\frac{1}{2}}}\|\omega(x, t)\|_{L^{2}\left(k D \times\left(-(k R)^{2}, 0\right)\right)} .
\end{aligned}
$$

One can also show that $\widetilde{\Gamma}(\widetilde{x}, \widetilde{t})=\widetilde{r} \widetilde{v_{\theta}}(\widetilde{x}, \widetilde{t})$ is a solution to $(1.2)$ and $\widetilde{\Omega}(\widetilde{x}, \widetilde{t})=\frac{\widetilde{\omega_{\theta}(\widetilde{x}, \widetilde{t})}}{\widetilde{r}}$ is a solution to (1.4) in the variables $(\widetilde{x}, \widetilde{t}) \in P_{1,4,1}$. We will do most of our computations on scaled cylinders.

Since $r v_{\theta}$ is scaling invariant, using the following result, we know that $\widetilde{r} \widetilde{v}_{\theta}$ is uniformly bounded for all time.

Proposition 2.1. ([3]) and [9]) Suppose $v$ is a smooth, axially symmetric solution of the three-dimensional Navier-Stokes equations with initial data $v_{0} \in L^{2}\left(\mathbb{R}^{3}\right)$. If $r v_{0, \theta} \in L^{p}\left(\mathbb{R}^{3}\right)$, then $r v_{\theta} \in L^{\infty}\left(0, T ; L^{p}\left(\mathbb{R}^{3}\right)\right)$. In particular, if $p=\infty$,

$$
\left|v_{\theta}(x, t)\right| \leq \frac{\left\|r v_{0, \theta}\right\|_{L^{\infty}\left(\mathbb{R}^{3}\right)}}{\sqrt{x_{1}^{2}+x_{2}^{2}}}
$$

\section{Proof of Theorem 1.2,}

During the proof, we are going to drop the "tilde" notation for all relevant quantities over a time when computations take place on the scaled cylinders. By the end, we will scale down to the original solution. Although this scaling seems merely a technical move that simplifies the computation, it is actually a key step that allows us to care out a dimension reduction argument mentioned earlier. In the region $P_{1,4,1}$ we do our analysis on (1.4):

$$
\Delta \Omega-(b \cdot \nabla) \Omega+\frac{2}{r} \frac{\partial \Omega}{\partial r}-\frac{\partial \Omega}{\partial t}+\frac{2 v_{\theta}}{r^{2}} \frac{\partial v_{\theta}}{\partial z}=0, \operatorname{div} b=0
$$

A flow chart for the argument to prove Theorem 1.2 is as follows:

Step 1: Energy Estimates by a refined cut-off function.

Step 2: Estimate drift term $(b \cdot \nabla) \Omega$ using methods similar to [21]. Use dimension reduction. Note this term is more singular than that allowed by standard theory.

Step 3: Estimate a term involving the cut-off.

Step 4: Estimate the term involving the directional derivative $\partial_{r}$ using a method similar to that in [4].

Step 5: Estimate the inhomogeneous term utilizing the bound in Proposition 2.1. 
Step 6: $L^{2}-L^{\infty}$ Estimate on Solutions to (1.4) via Moser's Iteration. Use dimension reduction.

$L^{2}-L^{\infty}$ Estimate on $\omega_{\theta}$ via re-scaling.

\section{Energy Estimates:}

Step 1: We use a revised cut-off function and the equation to obtain inequality (2.7) below.

Note that

$$
\Lambda \equiv\left\|v_{\theta}\right\|_{L^{\infty}\left(P_{1,4,1}\right)} \leq\left\|r v_{0, \theta}\right\|_{L^{\infty}\left(\mathbb{R}^{3}\right)}<\infty
$$

where we have used the hypothesis that $r v_{0, \theta} \in L^{\infty}\left(\mathbb{R}^{3}\right)$, the point-wise bound in Proposition 2.1, and the fact that $1<\sqrt{x_{1}^{2}+x_{2}^{2}}<4$. Let

$$
\bar{\Omega}_{+}(x, t)=\left\{\begin{array}{cc}
\Omega(x, t)+\Lambda & \Omega(x, t) \geq 0 \\
\Lambda & \Omega(x, t)<0 .
\end{array}\right.
$$

Note that $\bar{\Omega}_{+} \geq \Lambda$ and all derivatives of $\bar{\Omega}_{+}$on the set where $\Omega(x, t)<0$ are equal to zero. This function is also Lipschitz and $\Omega$ is smooth by assumption. At interfaces boundary terms upon integration by parts will cancel and so we can make sense of the calculations below. Direct computation yields, for $q>1$, that

$$
\Delta \bar{\Omega}_{+}^{q}-(b \cdot \nabla) \bar{\Omega}_{+}^{q}+\frac{2}{r} \partial_{r} \bar{\Omega}_{+}^{q}-\partial_{t} \bar{\Omega}_{+}^{q}=-\frac{q \bar{\Omega}_{+}^{q-1}}{r^{2}} \frac{\partial v_{\theta}^{2}}{\partial z}+q(q-1) \bar{\Omega}_{+}^{q-2}\left|\nabla \bar{\Omega}_{+}\right|^{2} .
$$

Let $\frac{5}{8} \leq \sigma_{2}<\sigma_{1} \leq 1$. Define

$$
\begin{aligned}
P\left(\sigma_{i}\right) & =\left\{(r, \theta, z):\left(5-4 \sigma_{i}\right)<r<4 \sigma_{i}, 0 \leq \theta \leq 2 \pi,|z|<4 \sigma_{i}\right\} \times\left(-\sigma_{i}^{2}, 0\right) \\
& =C\left(\sigma_{i}\right) \times\left(-\sigma_{i}^{2}, 0\right),
\end{aligned}
$$

for $i=1,2$. Here for convenience denote the space portion, which is a hollowed out cylinder, as $C\left(\sigma_{i}\right)$. Choose $\psi=\phi(y) \eta(s)$ to be a refined cut-off function satisfying

supp $\phi \subset C\left(\sigma_{1}\right) ; \phi(y)=1$ for all $y \in C\left(\sigma_{2}\right) ; 0 \leq \phi \leq 1 ;$

$$
\frac{|\nabla \phi|}{\phi^{\delta}} \leq \frac{c_{1}}{\sigma_{1}-\sigma_{2}} \text { for } \delta \in(0,1) \text { to be chosen later in the proof; }
$$

supp $\eta \subset\left(-\sigma_{1}^{2}, 0\right] ; \eta(s)=1$, for all $s \in\left[-\sigma_{2}^{2}, 0\right] ; 0 \leq \eta \leq 1$

$$
\left|\eta^{\prime}\right| \leq \frac{c_{2}}{\left(\sigma_{1}-\sigma_{2}\right)^{2}}
$$

Let $f=\bar{\Omega}_{+}^{q}$ and use $f \psi^{2}$ as a test function in (2.5) to get

$$
\begin{aligned}
\int_{P\left(\sigma_{1}\right)}( & \left.\Delta f-(b \cdot \nabla) f-\partial_{s} f+\frac{2}{r} \partial_{r} f\right) f \psi^{2} d y d s \\
& =\int_{P\left(\sigma_{1}\right)} q(q-1) \bar{\Omega}_{+}^{q-2}\left|\nabla \bar{\Omega}_{+}\right|^{2} f \psi^{2} d y d s-\int_{P\left(\sigma_{1}\right)} \frac{q \bar{\Omega}_{+}^{q-1}}{r^{2}} \frac{\partial v_{\theta}^{2}}{\partial z} f \psi^{2} d y d s \\
& =q(q-1) \int_{P\left(\sigma_{1}\right)} \bar{\Omega}_{+}^{-2}\left|\nabla \bar{\Omega}_{+}\right|^{2} f^{2} \psi^{2} d y d s-\int_{P\left(\sigma_{1}\right)} \frac{q \bar{\Omega}_{+}^{2 q-1}}{r^{2}} \frac{\partial v_{\theta}^{2}}{\partial z} \psi^{2} d y d s
\end{aligned}
$$




$$
\geq-\int_{P\left(\sigma_{1}\right)} \frac{q \bar{\Omega}_{+}^{2 q-1}}{r^{2}} \frac{\partial v_{\theta}^{2}}{\partial z} \psi^{2} d y d s .
$$

Integration by parts on the first term implies that

$$
\begin{aligned}
& \int_{P\left(\sigma_{1}\right)} \nabla\left(f \psi^{2}\right) \nabla f d y d s \\
& \leq \int_{P\left(\sigma_{1}\right)}\left(-b \cdot \nabla f\left(f \psi^{2}\right)-\partial_{s} f\left(f \psi^{2}\right)+\frac{2}{r} \partial_{r} f\left(f \psi^{2}\right)+\frac{q \bar{\Omega}_{+}^{2 q-1}}{r^{2}} \frac{\partial v_{\theta}^{2}}{\partial z} \psi^{2}\right) d y d s .
\end{aligned}
$$

A manipulation using the product rule shows that

$$
\int_{P\left(\sigma_{1}\right)} \nabla\left(f \psi^{2}\right) \nabla f d y d s=\int_{P\left(\sigma_{1}\right)}\left(|\nabla(f \psi)|^{2}-|\nabla \psi|^{2} f^{2}\right) d y d s .
$$

Thus,

$$
\begin{gathered}
\int_{P\left(\sigma_{1}\right)}|\nabla(f \psi)|^{2} d y d s \leq \int_{P\left(\sigma_{1}\right)}\left(-b \cdot \nabla f\left(f \psi^{2}\right)-\partial_{s} f\left(f \psi^{2}\right)+\frac{2}{r} \partial_{r} f\left(f \psi^{2}\right)\right. \\
\left.+\frac{q \bar{\Omega}_{+}^{2 q-1}}{r^{2}} \frac{\partial v_{\theta}^{2}}{\partial z} \psi^{2}+|\nabla \psi|^{2} f^{2}\right) d y d s .
\end{gathered}
$$

Integration by parts on the term involving the time derivative yields

$$
\begin{aligned}
& \int_{P\left(\sigma_{1}\right)}-\left(\partial_{s} f\right) f \psi^{2} d y d s=-\frac{1}{2} \int_{P\left(\sigma_{1}\right)} \partial_{s}\left(f^{2}\right) \psi^{2} d y d s \\
& =-\frac{1}{2}\left(\int_{C\left(\sigma_{1}\right)} f^{2} \psi^{2}(y, 0) d y-\int_{C\left(\sigma_{1}\right)} f^{2} \psi^{2}\left(y,-\sigma_{1}^{2}\right) d y\right)+\frac{1}{2} \int_{P\left(\sigma_{1}\right)} \partial_{s}\left(\psi^{2}\right) f^{2} d y d s .
\end{aligned}
$$

Our cut-off functions provide $\psi^{2}=(\phi \eta)^{2}, \eta(0)=1, \eta\left(-\sigma_{1}^{2}\right)=0$, and $0 \leq \phi \leq 1$. Thus,

$$
\begin{aligned}
\int_{P\left(\sigma_{1}\right)}-\left(\partial_{s} f\right) f \psi^{2} d y d s & =-\frac{1}{2} \int_{C\left(\sigma_{1}\right)} f^{2}(y, 0) \phi^{2}(y) d y+\int_{P\left(\sigma_{1}\right)} \phi^{2}\left(\eta \partial_{s} \eta\right) f^{2} d y d s \\
& \leq-\frac{1}{2} \int_{C\left(\sigma_{1}\right)} f^{2}(y, 0) \phi^{2}(y) d y+\int_{P\left(\sigma_{1}\right)}\left(\eta \partial_{s} \eta\right) f^{2} d y d s
\end{aligned}
$$

and so,

$$
\begin{aligned}
\int_{P\left(\sigma_{1}\right)} \mid \nabla & \left.(f \psi)\right|^{2} d y d s+\frac{1}{2} \int_{C\left(\sigma_{1}\right)} f^{2}(y, 0) \phi^{2}(y) d y \\
\leq & \int_{P\left(\sigma_{1}\right)}-b \cdot \nabla f\left(f \psi^{2}\right) d y d s+\int_{P\left(\sigma_{1}\right)}\left(\eta \partial_{s} \eta+|\nabla \psi|^{2}\right) f^{2} d y d s \\
& \quad+\int_{P\left(\sigma_{1}\right)} \frac{2}{r} \partial_{r} f\left(f \psi^{2}\right) d y d s+\int_{P\left(\sigma_{1}\right)} \frac{q \bar{\Omega}_{+}^{2 q-1}}{r^{2}} \frac{\partial v_{\theta}^{2}}{\partial z} \psi^{2} d y d s \\
:= & T_{1}+T_{2}+T_{3}+T_{4} .
\end{aligned}
$$

We will bound each of the terms on the right hand side in the next few steps. 
Step 2: In this step we find an upper bound for $T_{1}$, following an idea in [21], where a parabolic equation with a similar drift term is explored. The new input is that we can exploit the fact that in the space time domains of concern, all three dimensional integrals are equivalent to two dimensional ones. Therefore we can apply the 2 dimensional Sobolev inequality, which allows us to make gains. Another idea is to derive an a priori bound for the mean oscillation of the angular stream function by exploiting the structure of the equations.

Since $\operatorname{div} b=0$,

$$
\begin{aligned}
T_{1} & =\int_{P\left(\sigma_{1}\right)}-b \cdot(\nabla f)\left(f \psi^{2}\right) d y d s \\
& =\frac{1}{2} \int_{P\left(\sigma_{1}\right)}-b \psi^{2} \cdot \nabla\left(f^{2}\right) d y d s=\frac{1}{2} \int_{P\left(\sigma_{1}\right)} \operatorname{div}\left(b \psi^{2}\right) f^{2} d y d s \\
& =\frac{1}{2} \int_{P\left(\sigma_{1}\right)} \operatorname{div} b(\psi f)^{2} d y d s+\frac{1}{2} \int_{P\left(\sigma_{1}\right)} b \cdot \nabla\left(\psi^{2}\right) f^{2} d y d s \\
& =\int_{P\left(\sigma_{1}\right)} b \cdot(\nabla \psi) \psi f^{2} d y d s .
\end{aligned}
$$

In the following, we will often carry out computations on the following domains with 2 spatial dimension

$$
\begin{aligned}
& \bar{C}\left(\sigma_{1}\right)=\left\{(r, z) \mid(r, \theta, z) \in C\left(\sigma_{1}\right)\right\}, \\
& \bar{P}\left(\sigma_{1}\right)=\left\{(r, z, s) \mid(r, \theta, z, s) \in P\left(\sigma_{1}\right)\right\} .
\end{aligned}
$$

We will also use the following notations.

$$
\bar{y}=(r, z), \quad d \bar{y}=d r d z, \quad \text { if } \quad d y=r d r d z d \theta .
$$

Let $L_{\theta}$ be the angular component of the stream function in the cylindrical coordinates. It is well known that

$$
v_{r}=-\partial_{z} L_{\theta}, \quad v_{z}=\frac{1}{r} \partial_{r}\left(r L_{\theta}\right) .
$$

Note $L_{\theta}$ is also axially symmetric.

Let $a=a(t)$ be a function of time only, which will be chosen later. Using integration by parts and the divergence free property of $b=v_{r} e_{r}+v_{z} e_{z}$, we have

$$
\begin{aligned}
T_{1} & =\int_{P\left(\sigma_{1}\right)} b \cdot(\nabla \psi)\left(\psi f^{2}\right) d y d s \\
& =\int_{P\left(\sigma_{1}\right)}\left(v_{z} \partial_{z} \psi+v_{r} \partial_{r} \psi\right)\left(\psi f^{2}\right) d y d s \\
& \left.=2 \pi \int_{\bar{P}\left(\sigma_{1}\right)} \partial_{r}\left(r L_{\theta}-a\right)\left(\partial_{z} \psi\right)\left(\psi f^{2}\right) d r d z d s-2 \pi \int_{\bar{P}\left(\sigma_{1}\right)} \partial_{z}\left(r L_{\theta}-a\right)\left(\partial_{r} \psi\right)\left(\psi f^{2}\right)\right) d r d z d s \\
& \left.=-2 \pi \int_{\bar{P}\left(\sigma_{1}\right)}\left(r L_{\theta}-a\right) \partial_{r}\left[\left(\partial_{z} \psi\right)\left(\psi f^{2}\right)\right] d r d z d s+2 \pi \int_{\bar{P}\left(\sigma_{1}\right)}\left(r L_{\theta}-a\right) \partial_{z}\left[\left(\partial_{r} \psi\right)\left(\psi f^{2}\right)\right)\right] d r d z d s .
\end{aligned}
$$


From here, a routine calculation shows

$$
\begin{aligned}
T_{1} \leq & \frac{1}{8} \int_{P\left(\sigma_{1}\right)}|\nabla(\psi f)|^{2} d y d s \\
& \quad+C \frac{\sup _{t \in\left(-\sigma_{1}^{2}, 0\right)}\left(\left\|r L_{\theta}-a(t)\right\|_{L^{\infty}\left(\bar{C}\left(\sigma_{1}\right)\right)}^{2}+1\right)}{\left(\sigma_{1}-\sigma_{2}\right)^{2}} \int_{\bar{P}\left(\sigma_{1}\right)} f^{2} d \bar{y} d s .
\end{aligned}
$$

Here we have used the fact that $r$ is comparable to 1 in the region $P\left(\sigma_{1}\right)$ and therefore, the 2 dimensional volume element $d r d z$ is comparable to the three dimensional one $r d r d z d \theta$ for axially symmetric functions.

Choose a 2 dimensional cut-off function $\phi=\phi(r, z) \in C_{0}^{\infty}\left(\mathbb{R}^{2}\right)$ such that $\phi=1$ in $\bar{C}\left(\sigma_{1}\right)$, supp $\phi \in \bar{C}\left(9 \sigma_{1} / 8\right)$ and $0 \leq \phi \leq 1$, and $|\bar{\nabla} \phi|+\left|\Delta_{2} \phi\right| \leq C$. Observe that the region $\bar{C}\left(9 \sigma_{1} / 8\right)$ is at least $1 / 2$ unit away from the $z$ axis. Here and later in this section, $\bar{\nabla}=\left(\partial_{r}, \partial_{z}\right)$ is the 2 dimensional gradient, and $\Delta_{2}=\partial_{r}^{2}+\partial_{z}^{2}$ is the 2 dimensional Laplacian with respect to the $r$ and $z$ variables. In the 2 dimensional space of $(r, z)$, according to Hou-Li [7] Appendix,

$$
\begin{aligned}
\left\|r L_{\theta}-a(t)\right\|_{L^{\infty}\left(\bar{C}\left(\sigma_{1}\right)\right) \leq} & \left\|\left(r L_{\theta}-a(t)\right) \phi\right\|_{L^{\infty}\left(\bar{C}\left(9 \sigma_{1} / 8\right)\right)} \\
\leq & C\left(\left\|\bar{\nabla}\left(\left(r L_{\theta}-a(t)\right) \phi\right)\right\|_{L^{2}\left(\mathbb{R}^{2}\right)}+\left\|\left(r L_{\theta}-a(t)\right) \phi\right\|_{L^{2}\left(\mathbb{R}^{2}\right)}+1\right) \times \\
& {\left[\log \left(\left\|\Delta_{2}\left(\left(r L_{\theta}-a(t)\right) \phi\right)\right\|_{L^{2}\left(\mathbb{R}^{2}\right)}+\left\|\left(r L_{\theta}-a(t)\right) \phi\right\|_{L^{2}\left(\mathbb{R}^{2}\right)}+e\right)\right]^{\frac{1}{2}} } \\
\leq & C\left(\left\|\phi \bar{\nabla}\left(r L_{\theta}-a(t)\right)\right\|_{L^{2}\left(\mathbb{R}^{2}\right)}+\left\|\left(r L_{\theta}-a(t)\right)\right\|_{L^{2}\left(\bar{C}\left(9 \sigma_{1} / 8\right)\right)}+1\right) \times \\
& {\left[\log \left(\left\|\Delta_{2}\left(\left(r L_{\theta}-a(t)\right) \phi\right)\right\|_{L^{2}\left(\mathbb{R}^{2}\right)}+\left\|\left(r L_{\theta}-a(t)\right)\right\|_{L^{2}\left(\bar{C}\left(9 \sigma_{1} / 8\right)\right)}+e\right)\right]^{\frac{1}{2}} . }
\end{aligned}
$$

Choose $a(t)$ be the average of $r L_{\theta}(\cdot, t)$ on $\bar{C}\left(9 \sigma_{1} / 8\right)$ under the 2 dimensional volume element $d r d z$. Using the 2 dimension Poincaré inequality, we deduce

$$
\begin{aligned}
& \left\|r L_{\theta}-a(t)\right\|_{L^{\infty}\left(\bar{C}\left(\sigma_{1}\right)\right)} \leq C\left(\left\|\bar{\nabla}\left(r L_{\theta}\right)\right\|_{L^{2}\left(\bar{C}\left(9 \sigma_{1} / 8\right)\right)}+1\right) \times \\
& \quad\left[\log \left(\left\|\Delta_{2}\left(\left(r L_{\theta}-a(t)\right) \phi\right)\right\|_{L^{2}\left(\mathbb{R}^{2}\right)}+C\left\|\bar{\nabla}\left(r L_{\theta}\right)\right\|_{L^{2}\left(\bar{C}\left(9 \sigma_{1} / 8\right)\right)}+e\right)\right]^{\frac{1}{2}}
\end{aligned}
$$

Note that at any point $(x, t)$ we have,

$$
\left|\bar{\nabla}\left(r L_{\theta}\right)\right|^{2}=\left|\partial_{r}\left(r L_{\theta}\right)\right|^{2}+r^{2}\left|\partial_{z} L_{\theta}\right|^{2}=r^{2}\left[\left|\frac{1}{r} \partial_{r}\left(r L_{\theta}\right)\right|^{2}+\left|\partial_{z} L_{\theta}\right|^{2}\right]=r^{2}|b|^{2} .
$$


Also

$$
\begin{aligned}
& \Delta_{2}\left(\left(r L_{\theta}-a(t)\right) \phi\right)=\Delta_{2}\left(r L_{\theta}\right) \phi+2 \bar{\nabla}\left(r L_{\theta}\right) \cdot \bar{\nabla} \phi+\left(r L_{\theta}-a(t)\right) \Delta_{2} \phi \\
& \quad=\phi\left(\partial_{r}^{2}+\partial_{z}^{2}\right)\left(r L_{\theta}\right)+2 \bar{\nabla}\left(r L_{\theta}\right) \cdot \bar{\nabla} \phi+\left(r L_{\theta}-a(t)\right) \Delta_{2} \phi \\
& \quad=\phi r\left(\partial_{r}^{2} L_{\theta}+\partial_{z}^{2} L_{\theta}+\frac{2}{r} \partial_{r} L_{\theta}\right)+2 \bar{\nabla}\left(r L_{\theta}\right) \cdot \bar{\nabla} \phi+\left(r L_{\theta}-a(t)\right) \Delta_{2} \phi \\
& \quad=\phi r\left(\partial_{r}^{2} L_{\theta}+\partial_{z}^{2} L_{\theta}+\frac{1}{r} \partial_{r} L_{\theta}-\frac{1}{r^{2}} L_{\theta}\right)+\phi\left(\partial_{r} L_{\theta}+\frac{1}{r} L_{\theta}\right)+2 \bar{\nabla}\left(r L_{\theta}\right) \cdot \bar{\nabla} \phi+\left(r L_{\theta}-a(t)\right) \Delta_{2} \phi \\
& \quad=-\phi r w_{\theta}+\phi v_{z}+2 \bar{\nabla}\left(r L_{\theta}\right) \cdot \bar{\nabla} \phi+\left(r L_{\theta}-a(t)\right) \Delta_{2} \phi .
\end{aligned}
$$

Therefore, together with (2.12), we have,

$$
\left|\Delta_{2}\left(\left(r L_{\theta}-a(t)\right) \phi\right)\right| \leq\left|r w_{\theta}+v_{z}\right|+2 r|b|+C\left|\left(r L_{\theta}-a(t)\right)\right| .
$$

Using the 2 dimensional Poincaré inequality again, we find that

$$
\begin{aligned}
& \left\|\Delta_{2}\left(\left(r L_{\theta}-a(t)\right) \phi\right)\right\|_{L^{2}\left(\mathbb{R}^{2}\right)} \\
& \quad \leq C\left\|w_{\theta}\right\|_{L^{2}\left(\bar{C}\left(9 \sigma_{1} / 8\right)\right)}+C\|b\|_{L^{2}\left(\bar{C}\left(9 \sigma_{1} / 8\right)\right)}+C\left\|\left(r L_{\theta}-a(t)\right)\right\|_{L^{2}\left(\bar{C}\left(9 \sigma_{1} / 8\right)\right)} \\
& \quad \leq C\left\|w_{\theta}\right\|_{L^{2}\left(\bar{C}\left(9 \sigma_{1} / 8\right)\right)}+C\|b\|_{L^{2}\left(\bar{C}\left(9 \sigma_{1} / 8\right)\right)}+C\left\|\bar{\nabla}\left(r L_{\theta}\right)\right\|_{L^{2}\left(\bar{C}\left(9 \sigma_{1} / 8\right)\right)} \\
& \quad \leq C\left\|w_{\theta}\right\|_{L^{2}\left(\bar{C}\left(9 \sigma_{1} / 8\right)\right)}+2 C\|b\|_{L^{2}\left(\bar{C}\left(9 \sigma_{1} / 8\right)\right)} .
\end{aligned}
$$

Then from (2.11), we get

$$
\begin{aligned}
& \left\|r L_{\theta}-a(t)\right\|_{L^{\infty}\left(\bar{C}\left(\sigma_{1}\right)\right)} \\
& \leq C\left(\|v(\cdot, t)\|_{L^{2}\left(\bar{C}\left(9 \sigma_{1} / 8\right)\right)}+1\right)\left[\log \left(C\left\|w_{\theta}(\cdot, t)\right\|_{L^{2}\left(\bar{C}\left(9 \sigma_{1} / 8\right)\right)}+C\|v(\cdot, t)\|_{L^{2}\left(\bar{C}\left(9 \sigma_{1} / 8\right)\right)}+e\right)\right]^{\frac{1}{2}} .
\end{aligned}
$$

Then by (2.10), we have

$$
\begin{aligned}
& T_{1} \leq \frac{1}{8} \int_{P\left(\sigma_{1}\right)}|\nabla(\psi f)|^{2} d y d s \\
& +C \frac{\sup _{t \in\left(-\sigma_{1}^{2}, 0\right)}\left[\left(\|v(\cdot, t)\|_{L^{2}\left(\bar{C}\left(9 \sigma_{1} / 8\right)\right)}+1\right)^{2} \log \left(\left\|w_{\theta}(\cdot, t)\right\|_{L^{2}\left(\bar{C}\left(9 \sigma_{1} / 8\right)\right)}+\|v(\cdot, t)\|_{L^{2}\left(\bar{C}\left(9 \sigma_{1} / 8\right)\right)}+e\right)\right]}{\left(\sigma_{1}-\sigma_{2}\right)^{2}} \int_{\bar{P}\left(\sigma_{1}\right)} f^{2} d \bar{y} d s .
\end{aligned}
$$

Using the notation

$\bar{K}=\bar{K}(v, w) \equiv \sup _{t \in\left(-\sigma_{1}^{2}, 0\right)}\left[\left(\|v(\cdot, t)\|_{L^{2}\left(\bar{C}\left(9 \sigma_{1} / 8\right)\right)}+1\right) \log ^{1 / 2}\left(\left\|w_{\theta}(\cdot, t)\right\|_{L^{2}\left(\bar{C}\left(9 \sigma_{1} / 8\right)\right)}+\|v(\cdot, t)\|_{L^{2}\left(\bar{C}\left(9 \sigma_{1} / 8\right)\right)}+e\right)\right]$ we can write the last inequality as

$$
T_{1} \leq \frac{1}{8} \int_{P\left(\sigma_{1}\right)}|\nabla(\psi f)|^{2} d y d s+C \frac{\bar{K}^{2}(v, w)}{\left(\sigma_{1}-\sigma_{2}\right)^{2}} \int_{\bar{P}\left(\sigma_{1}\right)} f^{2} d \bar{y} d s .
$$


Step 3: The term $T_{2}$ is treated routinely. We use

$$
T_{2}=\int_{P\left(\sigma_{1}\right)}\left(\eta \partial_{s} \eta+|\nabla \psi|^{2}\right) f^{2} d y d s
$$

and properties of the cutoff,

$$
|\nabla \psi|^{2}=|\eta \nabla \phi|^{2} \leq\left(\frac{|\nabla \phi|}{\phi^{\delta}}\right)^{2} \leq \frac{c_{1}^{2}}{\left(\sigma_{1}-\sigma_{2}\right)^{2}}
$$

and

$$
\left|\eta \partial_{s} \eta\right| \leq\left|\partial_{s} \eta\right| \leq \frac{c_{2}}{\left(\sigma_{1}-\sigma_{2}\right)^{2}}
$$

to get

$$
\left|T_{2}\right| \leq \frac{C}{\left(\sigma_{1}-\sigma_{2}\right)^{2}} \int_{P\left(\sigma_{1}\right)} f^{2} d y d s \leq \frac{C}{\left(\sigma_{1}-\sigma_{2}\right)^{2}} \int_{\bar{P}\left(\sigma_{1}\right)} f^{2} d \bar{y} d s .
$$

Step 4: As we deal with $T_{3}=\int_{P\left(\sigma_{1}\right)} \frac{2}{r} \partial_{r} f\left(f \psi^{2}\right) d y d s$, we note we are assuming the integration takes place away from the singularity set of the solution to the axially symmetric Navier Stokes equations and away from the z-axis in general. Thus, all functions are bounded and smooth and $r$ varies between two positive constants. We also utilize the cylindrical coordinates of the axially symmetric case, and integration by parts:

$$
\begin{aligned}
T_{3} & =\int_{P\left(\sigma_{1}\right)} \frac{2}{r} \partial_{r} f\left(f \psi^{2}\right) d y d s=\int_{P\left(\sigma_{1}\right)} \frac{1}{r} \partial_{r}\left(f^{2}\right) \psi^{2} r d r d \theta d z d s \\
& =\int_{P\left(\sigma_{1}\right)} \partial_{r}\left(f^{2}\right) \psi^{2} d r d \theta d z d s=-\int_{P\left(\sigma_{1}\right)} \partial_{r}\left(\psi^{2}\right) f^{2} d r d \theta d z d s \\
& =-\int_{P\left(\sigma_{1}\right)} \frac{2}{r} \partial_{r} \psi\left(\psi f^{2}\right) r d r d \theta d z d s=-\int_{P\left(\sigma_{1}\right)} \frac{2}{r} \partial_{r}(\psi)\left(\psi f^{2}\right) d y d s \\
& =-\int_{P\left(\sigma_{1}\right)} \frac{2}{r} \overrightarrow{e_{r}} \cdot \nabla \psi\left(\psi f^{2}\right) d y d s .
\end{aligned}
$$

The Cauchy-Schwartz inequality then implies

$$
\left|T_{3}\right| \leq \int_{P\left(\sigma_{1}\right)} \frac{2}{r}|\nabla \psi| \psi f^{2} d y d s
$$

This yields

$$
\left|T_{3}\right| \leq \frac{C}{\left(\sigma_{1}-\sigma_{2}\right)} \int_{P\left(\sigma_{1}\right)} f^{2} d y d s \leq \frac{C}{\left(\sigma_{1}-\sigma_{2}\right)} \int_{\bar{P}\left(\sigma_{1}\right)} f^{2} d \bar{y} d s
$$

Step 5: Lastly, we work on the inhomogeneous term of (1.4), that is, $\frac{2 v_{\theta}}{r^{2}} \frac{\partial v_{\theta}}{\partial z}$, which produced the term $T_{4}$. Recall

$$
\Lambda=\left\|v_{\theta}\right\|_{L^{\infty}\left(P_{1,4,1}\right)} \leq\left\|r v_{0, \theta}\right\|_{L^{\infty}\left(\mathbb{R}^{3}\right)}<\infty,
$$


and that $\bar{\Omega}_{+}=\left\{\begin{array}{ll}\Omega+\Lambda & \Omega \geq 0 \\ \Lambda & \Omega<0\end{array}\right.$, thus $\bar{\Omega}_{+} \geq \Lambda$. Also, we have let $f=\bar{\Omega}_{+}^{q}$. Using integration by parts yields

$$
\begin{aligned}
T_{4} & =\int_{P\left(\sigma_{1}\right)} \frac{q \bar{\Omega}_{+}^{2 q-1}}{r^{2}} \frac{\partial v_{\theta}^{2}}{\partial z} \psi^{2} d y d s \\
& =-\int_{P\left(\sigma_{1}\right)} \frac{\partial}{\partial z}\left(\frac{\bar{\Omega}_{+}^{2 q} \psi^{2}}{\bar{\Omega}_{+}}\right) \frac{q}{r^{2}} v_{\theta}^{2} d y d s \\
& =-\int_{P\left(\sigma_{1}\right)} \frac{\partial}{\partial z}(f \psi)^{2} \frac{1}{\bar{\Omega}_{+}} \frac{q}{r^{2}} v_{\theta}^{2} d y d s+\int_{P\left(\sigma_{1}\right)}\left(\bar{\Omega}_{+}^{q} \psi\right)^{2} \frac{1}{\bar{\Omega}_{+}^{2}} \frac{\partial \bar{\Omega}_{+}}{\partial z} \frac{q}{r^{2}} v_{\theta}^{2} d y d s \\
& =-\int_{P\left(\sigma_{1}\right)} \frac{\partial}{\partial z}(f \psi)^{2} \frac{1}{\bar{\Omega}_{+}} \frac{q}{r^{2}} v_{\theta}^{2} d y d s \\
& \quad+\frac{1}{2} \int_{P\left(\sigma_{1}\right)} \frac{1}{\bar{\Omega}_{+}}\left[\frac{\partial\left(\bar{\Omega}_{+}^{2 q} \psi^{2}\right)}{\partial z}-\bar{\Omega}_{+}^{2 q} \frac{\partial \psi^{2}}{\partial z}\right] \frac{1}{r^{2}} v_{\theta}^{2} d y d s \\
& =-\int_{P\left(\sigma_{1}\right)} \frac{\partial}{\partial z}(f \psi)^{2} \frac{1}{\bar{\Omega}_{+}} \frac{q-(1 / 2)}{r^{2}} v_{\theta}^{2} d y d s-\frac{1}{2} \int_{P\left(\sigma_{1}\right)} \frac{1}{\bar{\Omega}_{+}} \bar{\Omega}_{+}^{2 q} \frac{\partial \psi^{2}}{\partial z} \frac{1}{r^{2}} v_{\theta}^{2} d y d s .
\end{aligned}
$$

Considering that $\frac{\left|v_{\theta}\right|}{\Lambda} \leq 1$, utilizing $\Lambda \leq \bar{\Omega}_{+}$, and $r=\sqrt{y_{1}^{2}+y_{2}^{2}} \geq 1$ for all $y \in P\left(\sigma_{1}\right)$, we continue by fixing $\epsilon_{3}>0$. Apply Young's inequality with exponents both being 2 to get

$$
\begin{aligned}
& \left|T_{4}\right| \leq \int_{P\left(\sigma_{1}\right)} 2 q\left|v_{\theta} \| f\right| \psi\left|\frac{\partial(f \psi)}{\partial z}\right| d y d s+\frac{c_{3}}{\sigma_{1}-\sigma_{2}} \int_{P\left(\sigma_{1}\right)} f^{2}\left|v_{\theta}\right| d y d s \\
& \leq \int_{P\left(\sigma_{1}\right)}\left|\frac{2 q \Lambda}{\left(2 \epsilon_{3}\right)^{\frac{1}{2}}} f \psi\right| \times\left|\left(2 \epsilon_{3}\right)^{\frac{1}{2}} \frac{\partial(f \psi)}{\partial z}\right| d y d s+\frac{c_{3} \Lambda}{\sigma_{1}-\sigma_{2}} \int_{P\left(\sigma_{1}\right)} f^{2} d y d s \\
& \leq \frac{c_{12} \Lambda^{2} q^{2}}{\epsilon_{3}} \int_{P\left(\sigma_{1}\right)} f^{2} d y d s+\epsilon_{3} \int_{P\left(\sigma_{1}\right)}|\nabla(f \psi)|^{2} d y d s+\frac{c_{3} \Lambda}{\sigma_{1}-\sigma_{2}} \int_{P\left(\sigma_{1}\right)} f^{2} d y d s .
\end{aligned}
$$

Thus

$$
\left|T_{4}\right| \leq \frac{1}{4} \int_{P\left(\sigma_{1}\right)}|\nabla(f \psi)|^{2} d y d s+C\left[\Lambda^{2} q^{2}+\frac{\Lambda}{\sigma_{1}-\sigma_{2}}\right] \int_{\bar{P}\left(\sigma_{1}\right)} f^{2} d \bar{y} d s
$$

Step 6: $L^{2}-L^{\infty}$ Estimate: An $L^{2}-L^{\infty}$ bound is derived using Moser's iteration. Recall inequality (2.7) from Step 1 and substitute the estimates for $T_{1}, T_{2}, T_{3}, T_{4}$ in (2.15), (2.16), (2.17), (2.18) respectively, we obtain

$$
\begin{aligned}
& \int_{P\left(\sigma_{1}\right)}|\nabla(f \psi)|^{2} d y d s+\frac{1}{2} \int_{C\left(\sigma_{1}\right)} f^{2}(y, 0) \phi^{2}(y) d y \\
& \leq \frac{3}{4} \int_{P\left(\sigma_{1}\right)}|\nabla(f \psi)|^{2} d y d s+C\left[\frac{C \bar{K}^{2}(v, w)}{\left(\sigma_{1}-\sigma_{2}\right)^{2}}+1+\Lambda^{2} q^{2}+\frac{1}{\left(\sigma_{1}-\sigma_{2}\right)^{2}}\right] \int_{\bar{P}\left(\sigma_{1}\right)} f^{2} d \bar{y} d s .
\end{aligned}
$$

Here $\bar{K}=\bar{K}(v, w)$ is defined in (2.14) . 
Consequently,

$$
\begin{array}{rl}
\int_{\bar{P}\left(\sigma_{1}\right)}|\bar{\nabla}(f \psi)|^{2} & d \bar{y} d s+\int_{\bar{C}\left(\sigma_{1}\right)} f^{2}(y, 0) \phi^{2}(y) d \bar{y} \\
& \leq \frac{C q^{2}}{\left(\sigma_{1}-\sigma_{2}\right)^{2}}\left(\bar{K}^{2}(v, w)\left(C_{1,4}\right)+\Lambda^{2}+1\right) \int_{\bar{P}\left(\sigma_{1}\right)} f^{2} d \bar{y} d s
\end{array}
$$

The last inequality follows since $q>1$ and $0<\sigma_{1}-\sigma_{2}<1$.

Next we will iterate the above energy type estimate. We will apply refined interpolation and embedding inequalities involving BMO functions. Applying Lemma 1 in Section 2 of [12, we know that

$$
\left\|(f \phi)^{2}\right\|_{L^{2}\left(\bar{C}\left(\sigma_{1}\right)\right)} \leq C\|f \phi\|_{L^{2}\left(\bar{C}\left(\sigma_{1}\right)\right)}\|f \phi\|_{B M O\left(\bar{C}\left(\sigma_{1}\right)\right)} .
$$

By the 2 dimensional Poincaré inequality, we also have

$$
\|f \phi\|_{B M O\left(\bar{C}\left(\sigma_{1}\right)\right)} \leq C\|\bar{\nabla}(f \phi)\|_{L^{2}} .
$$

These two inequalities imply that

$$
\|f \phi\|_{L^{4}\left(\bar{C}\left(\sigma_{1}\right)\right)} \leq C\|f \phi\|_{L^{2}\left(\bar{C}\left(\sigma_{1}\right)\right)}^{1 / 2}\|\bar{\nabla}(f \phi)\|_{L^{2}\left(\bar{C}\left(\sigma_{1}\right)\right)}^{1 / 2} .
$$

Consequently

$$
\begin{aligned}
\int_{-\sigma_{1}^{2}}^{0} \int_{\mathbb{R}^{2}}(f \psi)^{4} d \bar{y} d s & \\
& \leq C \sup _{-\sigma_{1}^{2} \leq s \leq 0}\left(\int_{\mathbb{R}^{2}}(f \psi)^{2} d \bar{y}\right) \int_{-\sigma_{1}^{2}}^{0} \int_{\mathbb{R}^{2}}|\bar{\nabla}(f \psi)|^{2} d \bar{y} d s .
\end{aligned}
$$

Substituting (2.19) into the right hand side of the above inequality, we deduce

$$
\int_{\bar{P}\left(\sigma_{2}\right)} f^{4} d \bar{y} d s \leq C\left[\frac{q^{2}}{\left(\sigma_{1}-\sigma_{2}\right)^{2}}\left[\bar{K}^{2}+\Lambda^{2}+1\right] \int_{\bar{P}\left(\sigma_{1}\right)} f^{2} d \bar{y} d s\right]^{2} .
$$

Here we have written $\bar{K}=\bar{K}(v, w)$ for simplicity. This shows, since $f=\bar{\Omega}_{+}^{q}$ by definition, that

$$
\int_{\bar{P}\left(\sigma_{2}\right)} \bar{\Omega}_{+}^{4 q} d \bar{y} d s \leq C\left[\frac{q^{2}}{\left(\sigma_{1}-\sigma_{2}\right)^{2}}\left[\bar{K}^{2}+\Lambda^{2}+1\right] \int_{\bar{P}\left(\sigma_{1}\right)} \bar{\Omega}_{+}^{2 q} d \bar{y} d s\right]^{2} .
$$

For $i=0,1,2, \ldots$, in (2.20), we take $q=2^{i}$, replace $\sigma_{1}$ by $\sigma_{i}=1-\Sigma_{j=1}^{i} 2^{-j-2}$ and $\sigma_{2}$ by $\sigma_{i+1}=1-\sum_{j=1}^{i+1} 2^{-j-2}$. Here we set $\Sigma_{j=1}^{i} 2^{-j-2}=0$ when $i=0$. Then

$$
\left(\int_{\bar{P}\left(\sigma_{i+1}\right)} \bar{\Omega}_{+}^{2^{i+2}} d \bar{y} d s\right)^{1 / 2} \leq c_{1} c_{2}^{i+1} 2^{2 i}\left(\bar{K}^{2}+\Lambda^{2}+1\right) \int_{\bar{P}\left(\sigma_{i}\right)} \bar{\Omega}_{+}^{2^{i+1}} d \bar{y} d s .
$$

After iteration, we obtain

$$
\left(\int_{\bar{P}\left(\sigma_{i+1}\right)} \bar{\Omega}_{+}^{2^{i+2}} d \bar{y} d s\right)^{\frac{1}{2^{i+1}}}
$$




$$
\leq c_{1}^{\sum \frac{1}{2^{j}}} c_{2}^{\sum \frac{j+1}{2^{j-1}}} 2^{2 \sum \frac{j-1}{2^{j-1}}}\left(\bar{K}^{2}+\Lambda^{2}+1\right)^{\sum \frac{1}{2^{j-1}}} \int_{\bar{P}_{1,4,1}} \bar{\Omega}_{+}^{2} d \bar{y} d s
$$

Note the sums in the exponents are all from $j=1$ to $j=i+1$. Let $i \rightarrow \infty$. All the exponential series converge and we deduce.

$$
\sup _{\bar{P}_{2,3,3 / 4}} \bar{\Omega}_{+}^{2} \leq C\left(\bar{K}^{2}+\Lambda^{2}+1\right)^{2} \int_{\bar{P}_{1,4,1}} \bar{\Omega}_{+}^{2} d \bar{y} d s .
$$

Repeating the argument on $\bar{\Omega}_{-}=\left\{\begin{array}{cc}-\Omega+\Lambda & \Omega \leq 0 \\ \Lambda & \Omega>0\end{array}\right.$, we see that

$$
\sup _{\bar{P}_{2,3,3 / 4}} \bar{\Omega}_{-}^{2} \leq C\left(\bar{K}^{2}+\Lambda^{2}+1\right)^{2} \int_{\bar{P}_{1,4,1}} \bar{\Omega}_{-}^{2} d \bar{y} d s \text {. }
$$

Thus

$$
\sup _{\bar{P}_{2,3,3 / 4}} \Omega^{2} \leq C\left(\bar{K}^{2}+\Lambda^{2}+1\right)^{2} \int_{\bar{P}_{1,4,1}}\left(\Omega^{2}+\Lambda^{2}\right) d \bar{y} d s .
$$

In the region $P_{1,4,1}$, the quantities $\Omega=\omega_{\theta} / r$ and $\omega_{\theta}$ are equivalent. Hence, the above implies

$$
\sup _{\bar{P}_{2,3,3 / 4}} \omega_{\theta}^{2} \leq C\left(\bar{K}^{2}+\Lambda^{2}+1\right)^{2} \int_{\bar{P}_{1,4,1}}\left(\omega_{\theta}^{2}+\Lambda^{2}\right) d \bar{y} d s .
$$

Re-scaling: We now recall that we omitted the "tildes" in the notation in the above computations. So what has actually been proven thus far is

$$
\sup _{(\widetilde{x}, \widetilde{t}) \in P_{2,3, \frac{3}{4}}} \widetilde{\omega}_{\theta}^{2}(\widetilde{x}, \widetilde{t}) \leq C(\bar{K}+\widetilde{\Lambda}+1)^{4}\left(\int_{P_{1,4,1}} \widetilde{\omega}_{\theta}^{2}(\widetilde{x}, \widetilde{t}) d \widetilde{x} d \widetilde{t}+\widetilde{\Lambda}^{2}\right),
$$

where $\widetilde{x}=\frac{x}{k}, \tilde{t}=\frac{t}{k^{2}}$ and from (2.14) with $\sigma_{1}=1$ that,

$$
\begin{aligned}
& \bar{K}=\bar{K}(\widetilde{v}, \widetilde{w}) \\
& \equiv \sup _{\widetilde{t} \in(-1,0)}\left[\left(\|\widetilde{v}(\cdot, \widetilde{t})\|_{L^{2}(\bar{C}(9 / 8))}+1\right) \log ^{1 / 2}\left(\left\|\widetilde{w}_{\theta}(\cdot, \widetilde{t})\right\|_{L^{2}(\bar{C}(9 / 8))}+\|\widetilde{v}(\cdot, \widetilde{t})\|_{L^{2}(\bar{C}(9 / 8))}+e\right)\right] \\
& \leq \sup _{\widetilde{t} \in(-1,0)}\left[\left(\|\widetilde{v}(\cdot, \widetilde{t})\|_{L^{2}(\bar{C}(0.5,5))}+1\right) \log ^{1 / 2}\left(\left\|\widetilde{w}_{\theta}(\cdot, \widetilde{t})\right\|_{L^{2}(\bar{C}(0.5,5))}+\|\widetilde{v}(\cdot, \widetilde{t})\|_{L^{2}(\bar{C}(0.5,5))}+e\right)\right] .
\end{aligned}
$$

Here we have used the fact that $\bar{C}(9 / 8) \subset \bar{C}(0.5,5)$ from the definition in (2.6), (2.1) and the 2 dimensional versions (2.8). We recall from the beginning of the section that

$$
\begin{aligned}
\|\widetilde{b}(\widetilde{x}, \widetilde{t})\|_{L^{\infty}\left(-1,0 ; L^{2}\left(C_{0.5,5}\right)\right)} & =k^{-\frac{1}{2}}\|b(x, t)\|_{L^{\infty}\left(-k^{2}, 0 ; L^{2}\left(C_{0.5 k, 5 k}\right)\right)}, \\
\|\widetilde{w}(\widetilde{x}, \widetilde{t})\|_{L^{\infty}\left(-1,0 ; L^{2}\left(C_{0.5,5}\right)\right)} & =k^{\frac{1}{2}}\|w(x, t)\|_{L^{\infty}\left(-k^{2}, 0 ; L^{2}\left(C_{0.5 k, 5 k}\right)\right)},
\end{aligned}
$$

and

$$
\|\widetilde{\omega}(\widetilde{x}, \widetilde{t})\|_{L^{2}\left(P_{1,4,1}\right)}=k^{-\frac{1}{2}}\|\omega(x, t)\|_{L^{2}\left(P_{k, 4 k, k}\right)} .
$$


Hence

$\bar{K} \leq \sup _{t \in\left[-k^{2}, 0\right]}\left(\frac{1}{k^{1 / 2}}\|b\|_{L^{2}\left(C_{1 k, 4 k}\right)}+1\right) \log ^{1 / 2}\left(k^{1 / 2}\left\|w_{\theta}(\cdot, t)\right\|_{L^{2}\left(C_{0.5 k, 5 k}\right)}+k^{-1 / 2}\|v(\cdot, t)\|_{L^{2}(C(0.5 k, 5 k))}+e\right)$

From [1], we have

$$
\left|\omega_{\theta}(x, t)\right| \leq \frac{C}{k^{5}}, \quad x \in C(0.5 k, 5 k)
$$

where $C$ only depends on the initial value. So, together with the energy inequality we have, for $k \in(0,1]$,

$$
\bar{K} \leq C\left(k^{-1 / 2}\|b\|_{L^{\infty}\left(-k^{2}, 0 ; L^{2}\left(C_{1 k, 4 k}\right)\right)}+1\right) \log ^{1 / 2}\left(\frac{1}{k}+e\right)
$$

where $C$ depends only on the initial value.

Also, we note the control on $\widetilde{\Lambda}$ is a scaling invariant quantity, since, by Proposition 2.1 , we have

$$
\begin{aligned}
\widetilde{\Lambda} & =\left(\sup _{P_{1,4,1}}\left|\widetilde{v_{\theta}}(\widetilde{x}, \widetilde{t})\right|\right) \leq\left(\left\|\widetilde{r} \widetilde{v_{\theta}}(\widetilde{x},-T)\right\|_{L^{\infty}\left(\mathbb{R}^{3}\right)}\right) \\
& =\left\|r v_{\theta}(x,-T)\right\|_{L^{\infty}\left(\mathbb{R}^{3}\right)}=\left\|r v_{0, \theta}\right\|_{L^{\infty}\left(\mathbb{R}^{3}\right) .}
\end{aligned}
$$

Substituting this and (2.23) into (2.22), we obtain, for $k \in(0,1)$ that

$$
\begin{gathered}
\sup _{(x, t) \in P_{2 k, 3 k, \frac{3 k}{4}} k^{4} \omega_{\theta}^{2}(x, t) \leq} \leq C\left(\left(k^{-1 / 2}\|b\|_{L^{\infty}\left(-k^{2}, 0 ; L^{2}\left(C_{1 k, 4 k}\right)\right)}+1\right) \log ^{1 / 2}\left(\frac{1}{k}+e\right)+\left\|r v_{0, \theta}\right\|_{L^{\infty}\left(\mathbb{R}^{3}\right)}+1\right)^{4} \\
\times\left(\int_{P_{k, 4 k, k}} k^{4} \omega_{\theta}^{2}(x, t) \frac{1}{k^{5}} d x d t+\left\|r v_{0, \theta}\right\|_{L^{\infty}\left(\mathbb{R}^{3}\right)}^{2}\right) \\
\leq \frac{C}{k^{3}}\left(\|b\|_{L^{\infty}\left(-k^{2}, 0 ; L^{2}\left(C_{1 k, 4 k}\right)\right)} \log ^{1 / 2}\left(\frac{1}{k}+e\right)+k^{1 / 2}\left\|r v_{0, \theta}\right\|_{L^{\infty}\left(\mathbb{R}^{3}\right)}+k^{1 / 2}\right)^{4} \\
\times\left(\left\|\omega_{\theta}\right\|_{L^{2}\left(P_{k, 4 k, k}\right)}^{2}+k\left\|r v_{0, \theta}\right\|_{L^{\infty}\left(\mathbb{R}^{3}\right)}^{2}\right) .
\end{gathered}
$$

Therefore,

$$
\begin{aligned}
& \left\|\omega_{\theta}(x, t)\right\|_{L^{\infty}\left(P_{2 k, 3 k, \frac{3 k}{4}}\right)} \leq \frac{C}{k^{7 / 2}}\left(\|b\|_{L^{\infty}\left(-k^{2}, 0 ; L^{2}\left(C_{1 k, 4 k}\right)\right)} \log ^{1 / 2}\left(\frac{1}{k}+e\right)+k^{1 / 2}\left\|r v_{0, \theta}\right\|_{L^{\infty}\left(\mathbb{R}^{3}\right)}+k^{1 / 2}\right)^{2} \\
& \times\left(\left\|\omega_{\theta}\right\|_{L^{2}\left(P_{k, 4 k, k}\right)}+\sqrt{k}\left\|r v_{0, \theta}\right\|_{L^{\infty}\left(\mathbb{R}^{3}\right)}\right) .
\end{aligned}
$$

This proves Theorem 1.2 .

\section{Velocity Bound, Proof of Theorem 1.1}

Based on the bound on $\omega_{\theta}$, now we prove the a priori velocity bound. In this section we ignore the time variable. Let $b=v_{r} e_{r}+v_{z} e_{z}$. Then taking the cylindrical curl of $b$ we get

$$
\operatorname{curl} b=\omega_{\theta} e_{\theta}
$$


Taking the curl on both sides of this equation and using the divergence free condition on $b$ to get curl $(\operatorname{curl} b)=-\Delta b$, we have

$$
-\Delta b=\operatorname{curl}\left(\omega_{\theta} e_{\theta}\right)
$$

Choose a smooth cutoff function $\phi$ with support contained in the ball $B_{2 r_{0}}=B\left(x, 2 r_{0}\right)$, $0 \leq \phi \leq 1$ in $B_{2 r_{0}}, \phi \equiv 1$ in $B_{\frac{r_{0}}{4}}$ and the following properties:

$$
|\nabla \phi| \leq \frac{C}{r_{0}} \quad, \quad|\Delta \phi| \leq \frac{C}{r_{0}^{2}}
$$

Then $\operatorname{supp}(\nabla \phi) \subset B_{2 r_{0}} \backslash B_{\frac{r_{0}}{4}}$, and we compute

$$
\begin{aligned}
\Delta(\phi b) & =\Delta \phi b+2 \nabla \phi \cdot \nabla b+\phi \Delta b \\
\Rightarrow \phi b & =\int_{B_{2 r_{0}}} \Gamma(x, y)\left(\Delta \phi b+2 \nabla \phi \cdot \nabla b-\phi \operatorname{curl}\left(\omega_{\theta} e_{\theta}\right)\right) d y \\
\phi b & =\int_{B_{2 r_{0}}} \Gamma(x, y) \Delta \phi b d y+2 \int_{B_{2 r_{0}}} \Gamma(x, y) \nabla \phi \cdot \nabla b d y-\int_{B_{2 r_{0}}} \Gamma(x, y) \phi \operatorname{curl}\left(\omega_{\theta} e_{\theta}\right) d y
\end{aligned}
$$

where $\Gamma(x, y)=\frac{c_{0}}{|x-y|}$ is the Green's function. After doing integration by parts, it is easy to see that, for all $p \geq 1$,

$$
\sup _{B_{r_{0}}(x)}|b| \leq C r_{0}^{-3 / p}\|b\|_{L^{p}\left(B_{2 r_{0}}(x)\right)}+C r_{0} \sup _{B_{2 r_{0}}(x)}\left|\omega_{\theta}\right| .
$$

Now we pick a point $x=\left(x_{1}, x_{2}, x_{3}\right)$ and let $r=\left|x^{\prime}\right|$ be the distance from $x$ to the $z$ axis again. We can assume $r \leq 1 / 2$ since we are only concerned with the bound near $z$ axis. Take $r_{0}=r^{1.5}|\ln r|^{-1 / 2}$. Then we can find $r /\left(2 r_{0}\right)=r^{-1 / 2}|\ln r|^{1 / 2} / 2$ (round up to nearest integer) many balls, which are disjoint, and which are generated by rotating $B\left(x, r_{0}\right)$ around the $z$ axis, such that, their union is contained in the torus around the curve

$$
\left\{y=\left(y_{1}, y_{2}, y_{3}\right) \mid \sqrt{y_{1}^{2}+y_{2}^{2}}=r, y_{3}=x_{3}\right\}
$$

with cross sections being 2 dimensional balls of radius $r$. Since the function $b$ is axially symmetric, the integral of $|b|^{2}$ on each of the ball is the same number. Therefore

$$
\|b\|_{L^{2}\left(B_{\left.2 r_{0}(x)\right)}\right.}^{2} \leq C r^{1 / 2}|\ln r|^{-1 / 2}\|b\|_{L^{2}\left(\mathbb{R}^{3}\right)}^{2} .
$$

Using this, we can now take $p=2$ in (3.1) and use the a priori bound on $\omega_{\theta}$ in Theorem 1.2 to deduce

$$
\begin{aligned}
|b(x, t)| & \leq C r_{0}^{-3 / 2} r^{1 / 2}|\ln r|^{-1 / 2}+C r_{0} r^{-7 / 2}|\ln r| \\
& =C r^{-9 / 4}|\ln r|^{3 / 4} r^{1 / 4}|\ln r|^{-1 / 4}+C r^{3 / 2}|\ln r|^{-1 / 2} r^{-7 / 2}|\ln r| \\
& =C r^{-2}|\ln r|^{1 / 2} .
\end{aligned}
$$

This completes the proof of Theorem 1.1. 


\section{Appendix: A Regularity CRIteria FOR $v_{z}$ ONLY}

In this section, we present 2 short results on equation (1.1), which may be of independent interest.

The first one is a critical regularity condition on the $z$ component of the velocity only. We note that a similar result under a little more assumption is given in Theorem 2.2 of the interesting paper [11. This result gives a mathematical explanation of the folklore belief that singularity can happen only if the vertical convection is high enough, in a tornado e.g.

Proposition 4.1. Let $v$ be a Leray-Hopf solution to (1.1) in $\mathbb{R}^{3} \times(0, \infty)$ such that $r v_{0, \theta} \in$ $L^{\infty}\left(\mathbb{R}^{3}\right)$. Suppose, for a given constant $C>0$, and all $x \in \mathbb{R}^{3}$ and $t \geq 0$,

$$
\left|v_{z}(x, t)\right| \leq \frac{C}{r}
$$

Then $v$ is regular for all time.

Proof. Recall from (2.9) the well known relation $v_{z}=\frac{1}{r} \partial_{r}\left(r L_{\theta}\right)$. Hence

$$
|| x^{\prime}\left|L_{\theta}(x, t)\right|=\left|\int_{0}^{\left|x^{\prime}\right|} \partial_{r}\left(r L_{\theta}\right) d r\right| \leq \int_{0}^{\left|x^{\prime}\right|}\left|r v_{z}\right| d r \leq C\left|x^{\prime}\right| .
$$

Here we just used the assumption on $v_{z}$. Therefore $L_{\theta}$ is a bounded function. Then from the main result in [15], we know that $v$ is regular for all time.

The next result is an a priori bound for the angular stream function, which scales in the same way as the energy estimate modulo a log term.

Proposition 4.2. Under the same assumption as Theorem 1.1, there exists a constant $C$, depending only on the initial data, such that,

$$
\left|L_{\theta}(x, t)\right| \leq \frac{C|\ln | x^{\prime}||^{1 / 2}}{\left|x^{\prime}\right|^{1 / 2}}, \quad\left|x^{\prime}\right| \leq \min \{1 / 2, R\}
$$

Proof. Recall from (2.13) the a priori bound for the scaled $L_{\theta}$ in a dyadic cube at time $t$ :

$$
\begin{aligned}
& \left\|r L_{\theta}-a(t)\right\|_{L^{\infty}\left(\bar{C}\left(\sigma_{1}\right)\right)} \\
& \leq C\left(\|v(\cdot, t)\|_{L^{2}\left(\bar{C}\left(9 \sigma_{1} / 8\right)\right)}+1\right)\left[\log \left(C\left\|w_{\theta}(\cdot, t)\right\|_{L^{2}\left(\bar{C}\left(9 \sigma_{1} / 8\right)\right)}+C\|v(\cdot, t)\|_{L^{2}\left(\bar{C}\left(9 \sigma_{1} / 8\right)\right)}+e\right)\right]^{\frac{1}{2}} .
\end{aligned}
$$


Here $a(t)$ is the average of the $r L_{\theta}(\cdot, t)$ in $\left.\bar{C}\left(9 \sigma_{1} / 8\right)\right)$ under the 2 dimensional volume element. After scaling as done at the end of Section 2, we find, for $k=\left|x^{\prime}\right|$ that,

$$
\begin{aligned}
\left|L_{\theta}(x, t)\right| & \leq \frac{C|\ln k|^{1 / 2}}{k^{1 / 2}}+C k^{-3} \int_{C_{0.5 k, 5 k}}\left|L_{\theta}\right| d y \\
& \leq \frac{C|\ln k|^{1 / 2}}{k^{1 / 2}}+C k^{-3}\left(\int_{C_{0.5 k, 5 k}}\left|L_{\theta}\right|^{6} d y\right)^{1 / 6} k^{5 / 2} \\
& \leq \frac{C|\ln k|^{1 / 2}}{k^{1 / 2}}+C k^{-1 / 2}\left(\int_{\mathbb{R}^{3}}\left|L_{\theta}\right|^{6} d y\right)^{1 / 6} \\
& \leq \frac{C|\ln k|^{1 / 2}}{k^{1 / 2}}+C k^{-1 / 2}\left(\int_{\mathbb{R}^{3}}|v|^{2} d y\right)^{1 / 2} .
\end{aligned}
$$

In the above we just used the 3 dimensional Sobolev inequality and the energy estimate for the velocity $v$. Thus $\left|L_{\theta}(x, t)\right| \leq \frac{C|\ln k|^{1 / 2}}{k^{1 / 2}}$ for $\left|x^{\prime}\right|=k \leq \min \{1 / 2, R\}$.

We remark that the energy estimate scales as $-1 / 2$ power as the distance. Since $L_{\theta}$ is scaling invariant, the bound in the proposition scales in the same way, modulo the log term.

\section{ACKNOWLEDGEMENTS}

Zhen Lei was in part supported by NSFC (grants No. 11171072, 11421061 and 11222107), SGST 09DZ2272900, Shanghai Talent Development Fund and Shanghai Municipal Dawn project. Q. S. Zhang gratefully acknowledges the supports by Siyuan Foundation through Nanjing University and by Simons Foundation. We should also thank Mr. Pan Xinghong for discussions on the problem. Finally, we are indebted to the anonymous referee who checked the paper very carefully and found an error in an earlier version of the paper.

\section{REFERENCES}

[1] Burke Loftus, Jennifer and Zhang, Qi S. A priori bounds for the vorticity of axially symmetric solutions to the Navier-Stokes equations. Adv. Differential Equations 15 (2010), no. 5-6, 531-560.

[2] L. Caffarelli, R. Kohn, and L. Nierenberg, Partial regularity of suitable weak solutions of the NavierStokes equations, Comm. Pure Appl. Math., 35 (1982), 771-831.

[3] Dongho Chae and Jihoon Lee, On the regularity of the axisymmetric solutions of the Navier-Stokes equations, Math. Z., 239 (2002), 645-671.

[4] Chiun-Chuan Chen, Robert M. Strain, Tai-Peng Tsai, and Horng-Tzer Yau, Lower bound on the blowup rate of the axisymmetric Navier-Stokes equations, Int. Math Res. Notices (2008), vol. 8, artical ID rnn016, $31 \mathrm{pp}$.

[5] _ Lower bound on th blow-up rate of the axisymmetric Navier-Stokes equations II,Comm. P.D.E., 34(2009), no. 1-3, 203-232.

[6] Thomas Y. Hou and Congming Li, Dynamic stability of the 3D axi-symmetric Navier-Stokes equations with swirl, Comm. Pure Appl. Math., 61 (2008) 661-697.

[7] Thomas Y. Hou and Congming Li, Global Well-Posedness of the Viscous Boussinesq Equations, Discrete and Continuous Dynamical Systems, Series A , 12(1), , 1-12, 2005.

[8] Thomas Y. Hou, Zhen Lei, and Congming Li, Global reuglarity of the 3D axi-symmetric Navier-Stokes equations with anisotropic data, Comm. P.D.E., 33 (2008), 1622-1637. 
[9] Jiri Neustupa and Milan Pokorny, An interior regularity criterion for an axially symmetric suitable weak solution to the Navier-Stokes equations, J. Math. Fluid Mech., 2 (2000), 381-399.

[10] Quansen Jiu and Zhouping Xin, Some regularity criteria on suitable weak solutions of the 3-D incompressible axisymmetric Navier-Stokes equations, Lectures on partial differential equations, New Stud. Adv. Math., vol. 2, Int. Press, Somerville, MA, 2003, pp. 119-139.

[11] Quansen Jiu and Zhouping Xin, On Liouville Theorems and Global Regularity to the 3-D Incompressible Axisymmetric Navier-Stokes Equations, arXiv:1501.02412

[12] Kozono, Hideo; Taniuchi, Yasushi, Bilinear estimates in BMO and the Navier-Stokes equations. Math. Z. 235 (2000), no. 1, 173-194.

[13] G. Koch, N. Nadirashvili, G. Seregin, and V. Sverak, Liouville theorems for the Navier-Stokes equations and applications, Acta Math. 203(2009), no. 1, 83-105.

[14] O. A. Ladyzhenskaya, Unique global solvability of the three-dimensional Cauchy problem for the NavierStokes equations in the presence of axial symmetry, Zap. Naucn. Sem. Leningrad. Otdel. Math. Inst. Steklov. (LOMI) 7 (1968), 155-177 (Russian).

[15] Z. Lei, and Q. S. Zhang, A Liouville Theorem for the Axially-symmetric Navier-Stokes Equations. Journal of Functional Analysis, 261 (2011), 2323-2345.

[16] - Structure of solutions of 3D Axi-symmetric Navier-Stokes Equations near Maximal Points. Pacific Journal of Mathematics, 254 (2011), no. 2, 335-344.

[17] S. Leonardi, J. Malek, J. Necas, and M. Porkorny, On axially symmetric flows in $\mathbb{R}^{3}$, Z. Anal. Anwendungen, 18 (1999), 639-649.

[18] G. Seregin and V. Sverak, On type I singularities of the local axi-symmetric solutions of the NavierStokes equations, Comm. P.D.E., 34(2009), no. 1-3, 171-201.

[19] Gang Tian and Zhouping Xin, One-point singular solutions to the Navier-Stokes equations, Topol. Methods Nonlinear Anal., 11 (1998), 135-145.

[20] M. R. Ukhovskii and V. I. Yudovich, Axially symmetric flows of ideal and viscous fluids filling the whole space, J. Appl. Math. Mech., 32 (1968), 52-61.

[21] Qi S. Zhang, A strong regularity result for parabolic equations, Comm. Math. Phys., 244 (2004), 245-260.

Z. L.: School of Mathematics, Fudan University, Shanghai 200433, P. R. China.

E. A. N. And Q.S.Z.: Department of Mathematics, University of California, Riverside, CA 92521 